\title{
Local boundedness of minimizers of anisotropic functionals
}

\author{
by \\ Andrea CIANCHI ${ }^{1}$ \\ Istituto di Matematica, Facoltà di Architettura, Università di Firenze, \\ Via dell’ Agnolo 14, 50122 Firenze, Italy \\ Manuscript received 16 October 1998
}

ABSTRACT. - The local boundedness of minimizers of functionals is proved under growth conditions depending on the full gradient.

(C) 2000 L'Association Publications de l'Institut Henri Poincaré. Published by Elsevier B.V. All rights reserved

\section{INTRODUCTION}

We are concerned with the local boundedness of local minimizers of integral functionals having the form

$$
J(u, \Omega)=\int_{\Omega} f(x, u, D u) \mathrm{d} x,
$$

where $\Omega$ is an open subset of $\mathbb{R}^{n}(n \geqslant 2)$ and $f: \Omega \times \mathbb{R} \times \mathbb{R}^{n} \rightarrow \mathbb{R}$ is a Carathéodory function.

In the classical theory of regularity (see e.g. $[5,8,11,14]$ ), as well as in more recent developments (including [1-3,6,7,10,12,13,15,16,18$25]$ ), the integrand $f$ is usually assumed to satisfy growth conditions

\footnotetext{
${ }^{1}$ E-mail: cianchi@ cesit1.unifi.it.
} 
depending on the gradient $D u$ only through its length $|D u|$, or through the sum of functions of the single partial derivatives $u_{x_{i}}, i=1, \ldots, n$.

The main novelty in the present paper is that bounds on $f$ are allowed involving functions (not necessarily of polynomial type) of the whole $D u$. An example of the functionals, not falling within the classes considered in the papers mentioned above, which we are able to deal with is

$$
\bar{J}(u, \Omega)=\int_{\Omega}\left[\left|u_{x_{i}}\right|^{p_{1}}+\left|u_{x_{1}}-u_{x_{2}}\right|^{p_{2}}+g\left(u_{x_{1}}, u_{x_{2}}\right)\right] \mathrm{d} x_{1} \mathrm{~d} x_{2},
$$

where $\Omega \subset \mathbb{R}^{2}, p_{1}, p_{2} \geqslant 1$ and $g$ is any bounded continuous function. The local boundedness of (possible) local minimizers of $\bar{J}$ can be discussed via Theorem 1, Section 2-see Example 3. Theorem 1 is a special case of the main result of this paper, which is contained in Theorem 2 of the same section. Let us point out that, even in standard situations, Theorem 2 slightly refines some of the results already available in the literature, in that it enables to include also certain borderline cases (Examples 1, 2, 4).

\section{MAIN RESULTS}

Our assumption on the integrand $f$ in (1.1) reads as follows:

$$
\begin{gathered}
A(\xi)-b(x) B(|s|)-a(x) \leqslant f(x, s, \xi) \\
\leqslant c(A(\xi)+b(x) B(|s|)+a(x))
\end{gathered}
$$

for $s \in \mathbb{R}, \xi \in \mathbb{R}^{n}$ and a.e. $x \in \Omega$. Here, $A: \mathbb{R}^{n} \rightarrow[0,+\infty)$ is a convex function such that

$$
A(0)=0, \quad \lim _{|\xi| \rightarrow+\infty} A(\xi)=+\infty
$$

and

$$
A(\xi)=A(-\xi) \quad \text { for every } \xi \in \mathbb{R}^{n} ;
$$

$B:[0,+\infty) \rightarrow[0,+\infty)$ is an increasing function; $a$ and $b$ are locally integrable nonnegative functions on $\Omega ; c$ is a constant $\geqslant 1$. Both $A$ and $B$ are required to satisfy the so-called $\Delta_{2}$-condition; namely, we assume that

$$
A(2 \xi) \leqslant k A(\xi) \quad \text { for } \xi \in \mathbb{R}^{n}
$$


and

$$
B(2 s) \leqslant k B(s) \text { for } s \geqslant 0,
$$

for some positive constant $k$.

A precise definition of a local minimizer of the functional $J$ in (1.1) involves the function $A_{M}:[0,+\infty) \rightarrow[0,+\infty)$ associated with $A$ by

$$
A_{M}(s)=\max _{|\xi|=s} A(\xi) \quad \text { for } s \geqslant 0
$$

In other words, $A_{M}(|\xi|)$ is the smallest radial function which dominates $A(\xi)$. It is easily verified that $A_{M}$ is a Young function, i.e., a convex function vanishing at zero. Notice also that $A_{M}$ satisfies the $\Delta_{2}$-condition whenever $A$ does.

A weakly differentiable function $u: \Omega \rightarrow \mathbb{R}$ will be called a local minimizer of $J$ if

$$
\int_{\Omega^{\prime}} A_{M}(|u|)+A(D u) \mathrm{d} x<\infty
$$

for every open set $\Omega^{\prime} \Subset \Omega$ and

$$
J(u, \operatorname{supp}(\phi)) \leqslant J(u+\phi, \operatorname{supp}(\phi))
$$

for every weakly differentiable function $\phi$ such that $\operatorname{supp}(\phi) \Subset \Omega$ and $\int_{\Omega} A_{M}(|\phi|)+A(D \phi) \mathrm{d} x<\infty$.

As shown by the counterexamples of [9,17] and [12], regularity of minimizers of $J$ cannot be expected, even in the simplest situation where $f$ is independent of $x$ and $s$, if $A$ is subject to the sole assumptions (2.2)-(2.4). Those counterexamples and the results of [7] suggest that a suitable additional assumption for minimizers to be locally bounded should amount to a bound for $A$ in terms of its Sobolev conjugate. An optimal Sobolev conjugate of $A$ is the function $A_{n}$ defined as follows (see [4]). Let $A_{\star}:[0,+\infty) \rightarrow[0,+\infty)$ be the increasing continuous function such that

$$
\left|\left\{\xi \in \mathbb{R}^{n}: A(\xi) \leqslant t\right\}\right|=\left|\left\{\xi \in \mathbb{R}^{n}: A_{\star}(|\xi|) \leqslant t\right\}\right| \text { for every } t \geqslant 0
$$

where $|\cdot|$ stands for Lebesgue measure; namely,

$$
A_{\star}(s)=\sup \left\{t:\left|\left\{\xi \in \mathbb{R}^{n}: A(\xi) \leqslant t\right\}\right|<C_{n} s^{n}\right\} \quad \text { for } s \geqslant 0,
$$


where $C_{n}$ is the measure of the $n$-dimensional unit ball. Then $A_{n}$ : $[0,+\infty) \rightarrow[0,+\infty]$ is defined as

$$
A_{n}=A_{\star} \circ H^{-1}, \quad \text { where } H(t)=\left(\int_{0}^{t}\left(\frac{r}{A_{\star}(r)}\right)^{n^{\prime}-1} \mathrm{~d} r\right)^{1 / n^{\prime}} \text { for } t \geqslant 0,
$$

$n^{\prime}=n /(n-1)$ and $H^{-1}$ is the left-continuous inverse of $H$. Note that, by the Brunn-Minkowski inequality, $\left|\left\{\xi \in \mathbb{R}^{n}: A(\xi) \leqslant t\right\}\right|^{1 / n}$ is a concave function of $t$. Thus $A_{\star}$ is a Young function and, since $H$ is concave and vanishes only at $0, A_{n}$ is a Young function as well.

In what follows we may always assume, without loss of generality, that the integral in the definition of $H$ in (2.10) is convergent-see Remark 2 below. Moreover, we shall suppose that

$$
\int^{+\infty}\left(\frac{s}{A_{\star}(s)}\right)^{n^{\prime}-1} \mathrm{~d} s=+\infty
$$

Indeed, if the integral in (2.11) converges, then any function $u$ satisfying (2.7) is automatically locally bounded-see Lemma 2, Section 2.

We are now in a position to state our regularity result. Because of its transparency, we give a separate statement in the basic case where the estimates in (2.1) are independent of $x$ and $s$, i.e., when

$$
A(\xi)-c \leqslant f(x, s, \xi) \leqslant c(A(\xi)+1)
$$

for $s \in \mathbb{R}, \xi \in \mathbb{R}^{n}$ and a.e. $x \in \Omega$.

THEOREM 1. - Assume that condition (2.12) is fulfilled for some A satisfying the above hypotheses. If a constant $k>0$ exists such that

$$
A(\xi) \leqslant A_{n}(k|\xi|)+k \quad \text { for every } \xi \in \mathbb{R}^{n},
$$

then any local minimizer of $J$ is locally bounded in $\Omega$.

In the general case, a balance has to be imposed between the degrees of summability of $a$ and $b$, and the growths of $A, B$ and $A_{n}$. Such a balance involves the lower index at infinity of the functions $A_{\star}$ and $A_{M}+B$. Recall that such an index is defined for an increasing function $\Phi:(0,+\infty) \rightarrow(0,+\infty)$ as

$$
i_{\infty}(\Phi)=\lim _{\lambda \rightarrow+\infty} \frac{\log \left(\liminf \operatorname{in}_{s \rightarrow+\infty} \frac{\Phi(\lambda s)}{\Phi(s)}\right)}{\log \lambda} .
$$


THEOREM 2. - Assume that condition (2.1) is fulfilled for some A and $B$ satisfying the above hypotheses. Suppose that:

(i) $b \in L_{\text {loc }}^{\beta}(\Omega)$ for some $\beta \in(1, \infty]$ such that $\beta>n / i_{\infty}\left(A_{M}+B\right)$ and

$$
(A(\xi)+B(|\xi|))^{\beta^{\prime}} \leqslant A_{n}(k|\xi|)+k \quad \text { for } \xi \in \mathbb{R}^{n},
$$

for some positive constant $k$;

(ii) $a \in L_{\text {loc }}^{\alpha}(\Omega)$ for some $\alpha>n / i_{\infty}\left(A_{\star}\right)$.

Then any local minimizer of $J$ is locally bounded in $\Omega$.

Remark 1. - Assumption (2.11) implies, in particular, that $i_{\infty}\left(A_{\star}\right) \leqslant n$ (see Proposition 1, Section 3).

Remark 2. - Given any radial convex function $\Lambda$ vanishing at 0 and satisfying the $\Delta_{2}$-condition, we may assume, without loss of generality, that $A(\xi)=\Lambda(\xi)$ if $|\xi|$ is small enough. Actually, it is not difficult to see that there exist positive constants $c_{1}, c_{2}, s_{0}$ and $t_{0}$ such that, if $\bar{A}(\xi)$ is the function which equals $\Lambda(\xi)$ for $|\xi| \leqslant s_{0}$, agrees with $c_{1} A(\xi)-c_{2}$ in $\left\{\xi \in \mathbb{R}^{n}: A(\xi)>t_{0}\right\}$ and is extended by convexity otherwise, then $\bar{A}$ is a convex function fulfilling (2.2)-(2.4) and

$$
\frac{1}{c_{1}}\left(\bar{A}(\xi)+c_{2}\right)-t_{0} \leqslant A(\xi) \leqslant \frac{1}{c_{1}}\left(\bar{A}(\xi)+c_{2}\right)+t_{0} \quad \text { for } \xi \in \mathbb{R}^{n}
$$

Thus, $f$ satisfies (2.1) with $A(\xi)$ replaced by $\bar{A}(\xi) / c_{1}$ and with $a(x)$ replaced by $\bar{a}(x)=a(x)+t_{0}+c_{2} / c_{1}$; moreover, conditions (i)-(ii) of Theorem 2 with $A$ and $a$ replaced by $\bar{A} / c_{1}$ and $\bar{a}$, respectively, are equivalent to the original ones.

Example 1.-Consider the classical case where $A(\xi)=|\xi|^{p}$ and $B(s)=s^{q}$. In this case, $A_{n}(s)$ is equivalent to $s^{p^{*}}$, where $p^{*}=n p /(n-$ $p)$, if $1 \leqslant p<n$, and is equivalent near infinity to $\exp \left(s^{n^{\prime}}\right)-1$ if $p=n$. Recall that two functions $\Phi_{1}, \Phi_{2}:[0,+\infty) \rightarrow[0,+\infty)$ are called equivalent if there exist positive constants $c_{1}$ and $c_{2}$ such that $\Phi_{1}\left(c_{1} s\right) \leqslant$ $\Phi_{2}(s) \leqslant \Phi_{1}\left(c_{2} s\right)$, for $s \geqslant 0$, and are called equivalent near infinity if the same inequalities hold for large $s$. Thus, when $1 \leqslant p<n$, Theorem 2 tells us that any local minimizer of $J$ is locally bounded, provided that $0<q \leqslant p^{*}, b \in L_{\mathrm{loc}}^{\beta}(\Omega)$ with $\beta=p^{*} /\left(p^{*}-q\right)$ or $\beta>n / p$ according to whether $p<q$ or $p \geqslant q$, and $a \in L_{\text {loc }}^{\alpha}(\Omega)$ for some $\alpha>n / p$. If $p=n$, the same conclusion is true for every $q>0$, provided that $b \in L_{\text {loc }}^{\beta}(\Omega)$ 
and $a \in L_{\text {loc }}^{\alpha}(\Omega)$ for some $\beta, \alpha>1$. This reproduces the result of [10] and shows that the limiting values $q=p^{*}$ and $\beta=p^{*} /\left(p^{*}-q\right)$ are allowed.

Example 2. - Assume, more generally, that $A$ is just radial, so that $A(\xi)=A_{\star}(|\xi|)=A_{M}(|\xi|)$. Since $H^{-1}$ grows more than linearly at infinity, condition (2.13) is always fulfilled; thus, the results on boundedness contained in $[21,22]$ are recovered by Theorem 1 and extended by Theorem 2. The latter also improves a result from [16].

Example 3. - Let us take into account the functional $\bar{J}$ defined by (1.2). An estimate of type (2.12) obviously holds with

$$
A(\xi)=\left|\xi_{1}\right|^{p_{1}}+\left|\xi_{1}-\xi_{2}\right|^{p_{2}}
$$

where $\xi=\left(\xi_{1}, \xi_{2}\right) \in \mathbb{R}^{2}$. The straight lines $\xi_{1}= \pm t^{1 / p_{1}}$ and $\xi_{2}=\xi_{1} \pm t^{1 / p_{2}}$ are tangent to the (convex) level set $\left\{\xi \in \mathbb{R}^{2}: A(\xi) \leqslant t\right\}$ for every $t \geqslant 0$. Consequently,

$$
2 t^{1 / p_{1}+1 / p_{2}} \leqslant\left|\left\{\xi \in \mathbb{R}^{2}: A(\xi) \leqslant t\right\}\right| \leqslant 4 t^{1 / p_{1}+1 / p_{2}} \text { for } t \geqslant 0 .
$$

Thus, $A_{\star}(s)$ is equivalent to $s^{2 p_{1} p_{2} /\left(p_{1}+p_{2}\right)}$, so that $A_{n}(s)$ is equivalent to $s^{2 p_{1} p_{2} /\left(p_{1}+p_{2}-p_{1} p_{2}\right)}$ if $p_{1} p_{2}<p_{1}+p_{2}$, and is equivalent near infinity to $\exp \left(s^{2}\right)-1$ if $p_{1} p_{2}=p_{1}+p_{2}$. Theorem 1 (and the remark about condition (2.11)) tell us that the local minimizers of $\bar{J}$ are locally bounded either if $p_{1} p_{2} \geqslant p_{1}+p_{2}$ or if $p_{1} p_{2}<p_{1}+p_{2}$ and $\min \left\{p_{1}, p_{2}\right\} \geqslant$ $\max \left\{p_{1}, p_{2}\right\} /\left(1+\max \left\{p_{1}, p_{2}\right\}\right)$.

Consider now the special case where $A$ has the form

$$
A(\xi)=\sum_{i=1}^{n} A_{i}\left(\left|\xi_{i}\right|\right)
$$

where $\xi=\left(\xi_{1}, \ldots, \xi_{n}\right)$ and $A_{i}$ are Young functions. In this case, $A_{\star}$ is equivalent to the function $\bar{A}:[0,+\infty) \rightarrow[0,+\infty)$ whose (rightcontinuous) inverse is defined by

$$
\bar{A}^{-1}(t)=\left(\prod_{i=1}^{n} A_{i}^{-1}(t)\right)^{1 / n} \quad \text { for } t \geqslant 0 .
$$

Indeed, since $\left\{\xi \in \mathbb{R}^{n}: A(\xi) \leqslant t\right\}$ is a convex set containing the points $\left(0, \ldots, 0, \pm A_{i}^{-1}(t), 0, \ldots, 0\right)$ and contained in the parallelepiped 
bounded by the hyperplanes $\xi_{i}= \pm A_{i}^{-1}(t)$, then

$$
\frac{2^{n}}{n !} \prod_{i=1}^{n} A_{i}^{-1}(r) \leqslant\left|\left\{\xi \in \mathbb{R}^{n}: A(\xi) \leqslant t\right\}\right| \leqslant 2^{n} \prod_{i=1}^{n} A_{i}^{-1}(r) .
$$

Hence the equivalence of $A_{\star}$ and $\bar{A}$ follows. Moreover, the function $A_{n}$ is easily seen to be equivalent to the function $\bar{A}_{n}$ defined as in (2.10), but with $A_{\star}$ replaced by $\bar{A}$. Notice also that $A_{M}(s)$ is equivalent to $\max _{i} A_{i}(s)$ (and to $\sum_{i=1}^{n} A_{i}(s)$ ). Theorem 2, combined with these considerations, yields the following corollary.

Corollary. - Assume that condition (2.1) is fulfilled for some A having the form (2.16) and that the Young funtions $A_{i}$ and the function $B$ satisfy the $\Delta_{2}$-condition. Suppose that:

(i) $b \in L_{\mathrm{loc}}^{\beta}(\Omega)$ for some $\beta \in(1, \infty]$ such that $\beta>n / i_{\infty}(B+$ $\max _{i} A_{i}$ ) and

$$
\left(\max _{i} A_{i}\left(\left|\xi_{i}\right|\right)+B(|\xi|)\right)^{\beta^{\prime}} \leqslant \bar{A}_{n}(k|\xi|)+k \quad \text { for } \xi \in \mathbb{R}^{n}
$$

for some positive constant $k$;

(ii) $a \in L_{\text {loc }}^{\alpha}(\Omega)$ for some $\alpha>n / i_{\infty}(\bar{A})$.

Then any local minimizer of $J$ is locally bounded in $\Omega$.

Obviously, $\max _{i} A_{i}$ can be replaced by $\sum_{i=1}^{n} A_{i}$ in assumption (i) of the Corollary.

Let us mention that a result in the same direction as the Corollary is contained in [13].

Example 4. - Let $A_{i}(s)=s^{p_{i}}$ for some $p_{i} \geqslant 1, i=1, \ldots, n$, and $B(s)=s^{q}$. Then $\bar{A}(s)=s^{\bar{p}}$, where $1 / \bar{p}=(1 / n) \sum_{i=1}^{n}\left(1 / p_{i}\right)$, so that $\bar{A}_{n}(s)$ is equivalent to $s^{\bar{p}^{*}}$ if $\bar{p}<n$, and is equivalent near infinity to $\exp \left(s^{n^{\prime}}\right)-1$ if $\bar{p}=n$. Set $m=\max \left\{p_{1}, \ldots, p_{n}, q\right\}$. When $\bar{p}<n$, the Corollary yields the local boundedness of local minimizers of $J$ if $m \leqslant$ $\bar{p}^{*}, b \in L_{\mathrm{loc}}^{\beta}(\Omega)$ with $\beta=\bar{p}^{*} /\left(\bar{p}^{*}-m\right)$ or $\beta>n / \bar{p}$ according to whether $\bar{p}<m$ or $\bar{p} \geqslant m$, and $a \in L_{\text {loc }}^{\alpha}(\Omega)$ for some $\alpha>n / \bar{p}$. When $\bar{p}=n$, the same conclusion holds for every $q>0$, provided that $b \in L_{\text {loc }}^{\alpha}(\Omega)$ and $a \in L_{\text {loc }}^{\alpha}(\Omega)$ for some $\beta, \alpha>1$. This example includes Theorem 3.1 of [7], where $b(x)$ was taken $\equiv 1$. 


\section{SOBOLEV AND CACCIOPPOLI INEQUALITIES}

Basic tools in the proof of Theorem 2 are a general anisotropic version of the Sobolev inequality and a Caccioppoli type inequality for local minimizers of $J$.

The Sobolev inequality we allude to is stated in the following theorem.

THEOREM. - Let A be any convex function satisfying (2.2)-(2.3) and making the integral in (2.10) converge. Then there exists a constant $c(n)$, depending only upon $n$, such that

$$
\int_{\mathbb{R}^{n}} A_{n}\left(\frac{|u(x)|}{c(n)\left(\int_{\mathbb{R}^{n}} A(D u) \mathrm{d} y\right)^{1 / n}}\right) \mathrm{d} x \leqslant \int_{\mathbb{R}^{n}} A(D u) \mathrm{d} x
$$

for every real-valued weakly differentiable function $u$ on $\mathbb{R}^{n}$ decaying to 0 at infinity, in the sense that $\left|\left\{x \in \mathbb{R}^{n}:|u(x)|>t\right\}\right|<+\infty$ for every $t>0$. The function $A_{n}$ is optimal, in the sense that if (3.1) holds with $A_{n}$ replaced by any Young function $A_{0}$ for every A satisfying (2.2)-(2.3) and with prescribed $A_{\star}$, then the integral in (2.10) must converge and there exists $c>0$ such that $A_{0}(s) \leqslant A_{n}(c s)$ for $s \geqslant 0$.

A proof of this theorem can be found in [4]. We shall need the consequences contained in Lemmas 1 and 2 below.

LEMMA 1. - Let A be any convex function satisfying (2.2)-(2.3) and making the integral in (2.10) converge. Let $\Phi, \Psi:[0,+\infty) \rightarrow[0,+\infty)$ be increasing functions such that

$$
\Phi(\lambda s) \leqslant \Psi(\lambda) \Phi(s) \quad \text { for } \lambda, s \geqslant 0 .
$$

Assume that

$$
\Phi(s) \leqslant A_{n}(s) \quad \text { for } s \geqslant 0 .
$$

Then

$$
\int_{\mathbb{R}^{n}} \Phi(k|u(x)|) \mathrm{d} x \leqslant \Psi\left(k c(n)\left(\int_{\mathbb{R}^{n}} A(D u) \mathrm{d} x\right)^{1 / n}\right) \int_{\mathbb{R}^{n}} A(D u) \mathrm{d} x
$$

for every $k>0$ and every real-valued weakly differentiable function $u$ on $\mathbb{R}^{n}$ decaying to 0 at infinity. Here, $c(n)$ is the constant appearing in (3.1).

The derivation of Lemma 1 from inequality (3.1) is straightforward. Let us notice that the existence of a function $\Psi$ rendering (3.2) true 
is equivalent to the $\Delta_{2}$-condition for $\Phi$. This is a consequence of Proposition 1 below containing standard results relating the growth of an increasing function $\Phi:(0,+\infty) \rightarrow(0,+\infty)$ with its indices $i(\Phi), I(\Phi), i_{\infty}(\Phi)$ and $I_{\infty}(\Phi)$. Here, $i_{\infty}(\Phi)$ is the number defined by (2.14); $I_{\infty}(\Phi)$ is defined similarly, but with $\liminf _{s \rightarrow+\infty}$ replaced by limsup $\operatorname{su+\infty }_{s \rightarrow+} ; i(\Phi)$ and $I(\Phi)$ are defined as $i_{\infty}(\Phi)$ and $I_{\infty}(\Phi)$, save that $\liminf _{s \rightarrow+\infty}$ and limsup $\sin _{s \rightarrow+\infty}$ are replaced by $\inf _{s>0}$ and $\sup _{s>0}$, respectively. Obviously, $0 \leqslant i(\Phi) \leqslant i_{\infty}(\Phi) \leqslant I_{\infty}(\Phi) \leqslant I(\Phi) \leqslant+\infty$; in particular, if $\Phi$ is a Young function, then 0 can be replaced by 1 in these inequalities.

PROPOSITION 1. - Let $\Phi:(0,+\infty) \rightarrow(0,+\infty)$ be any increasing function.

(i) $\Phi$ satisfies the $\Delta_{2}$-condition if and only if $I(\Phi)<\infty$. In this case, for every $\delta>0$ there exists a constant $C>0$ such that

$$
\begin{aligned}
& \frac{1}{C} \min \left\{\lambda^{i(\Phi)-\delta}, \lambda^{I(\Phi)+\delta}\right\} \Phi(s) \\
& \quad \leqslant \Phi(\lambda s) \leqslant C \max \left\{\lambda^{i(\Phi)-\delta}, \lambda^{I(\Phi)+\delta}\right\} \Phi(s) \text { for } \lambda, s>0 .
\end{aligned}
$$

(ii) If $I_{\infty}(\Phi)<\infty$, then for every $\delta>0$ there exist positive constants $C$ and $s_{0}$ such that

$$
\begin{aligned}
& \frac{1}{C} \lambda^{i_{\infty}(\Phi)-\delta} \Phi(s) \\
& \quad \leqslant \Phi(\lambda s) \leqslant C \lambda^{I_{\infty}(\Phi)+\delta} \Phi(s) \text { for } s \geqslant s_{0} \text { and } \lambda \geqslant 1 .
\end{aligned}
$$

In what follows, $B_{R}$ will denote a ball of radius $R$ in $\mathbb{R}^{n}$.

LEMMA 2. - Let $A$ be any convex function fulfilling (2.2)-(2.4) and let $u$ be any weakly differentiable function on $\Omega$ such that $\int_{\Omega^{\prime}} A_{M}(|u|)+$ $A(D u) \mathrm{d} x<\infty$ for every open set $\Omega^{\prime} \Subset \Omega$.

(i) If $\int^{+\infty}\left(s / A_{\star}(s)\right)^{n^{\prime}-1} \mathrm{~d} s<+\infty$, then $u$ is locally bounded.

(ii) If $\int^{+\infty}\left(s / A_{\star}(s)\right)^{n^{\prime}-1} \mathrm{~d} s=+\infty$, then

$$
\int_{B_{R}} A_{n}(k|u(x)|) \mathrm{d} x<+\infty
$$

for every $k>0$ and every ball $B \Subset \Omega$.

Proof. - Let $k$ be any positive number. Fix any ball $B_{R} \Subset \Omega$ and let $B_{\bar{R}}$ be a ball, concentric with $B_{R}$, such that $B_{R} \Subset B_{\bar{R}} \Subset \Omega$. Let $\eta$ be any real-valued smooth function on $\mathbb{R}^{n}$ such that $0 \leqslant \eta(x) \leqslant 1$ for 
every $x \in \mathbb{R}^{n}, \eta \equiv 1$ in $B_{R}, \eta \equiv 0$ outside $B_{\bar{R}}$ and $|D \eta| \leqslant 2 /(\bar{R}-R)$ everywhere. Given any $t>0$, we have

$$
\begin{aligned}
\int_{B_{R}} A_{n}(k|u|) \mathrm{d} x \leqslant & \int_{B_{\bar{R}}} A_{n}(k|u| \eta) \mathrm{d} x \\
= & \int_{\{|u| \eta>t\}} A_{n}(k|u| \eta) \mathrm{d} x+\int_{\{|u| \eta \leqslant t\}} A_{n}(k|u| \eta) \mathrm{d} x \\
\leqslant & \frac{1}{2} \int_{\{|u| \eta>t\}} A_{n}(2 k(|u| \eta-t)) \mathrm{d} x+\frac{1}{2} \int_{\{|u| \eta>t\}} A_{n}(2 k t) \mathrm{d} x \\
& +\int_{\{|u| \eta \leqslant t\}} A_{n}(k|u| \eta) \mathrm{d} x,
\end{aligned}
$$

since $A_{n}$ is convex. The convexity of $A$, our assumptions on $\eta$ and Proposition 1 applied to $A_{M}$ easily imply that there exists a positive constant $C$ (independent of $u$ and $t$ ), such that

$$
\int_{\{|u| \eta>t\}} A(D[|u| \eta]) \mathrm{d} x \leqslant C\left(\int_{\{|u| \eta>t\}} A(D u) \mathrm{d} x+\int_{\{|u| \eta>t\}} A_{M}(|u|) \mathrm{d} x\right) .
$$

By (2.7), the right-hand side of the last inequality is finite. Thus, $t$ can be chosen so large that

$$
2 k c(n)\left(\int_{\{|u| \eta>t\}} A(D[|u| \eta]) \mathrm{d} x\right)^{1 / n} \leqslant 1,
$$

where $c(n)$ is the constant of inequality (3.1). Since $A_{n}$ is a Young function, $A_{n}(\lambda s) \leqslant \lambda A_{n}(s)$ for $0 \leqslant \lambda \leqslant 1$ and $s \geqslant 0$. Thus, inequalities (3.1) and (3.9) imply that

$$
\begin{aligned}
& \int_{\{|u| \eta>t\}} A_{n}(2 k(|u| \eta-t)) \mathrm{d} x \\
& \leqslant 2 k c(n)\left(\int_{\{|u| \eta>t\}} A(D[|u| \eta]) \mathrm{d} x\right)^{1 / n} \\
& \quad \times \int_{\{|u| \eta>t\}} A_{n}\left(\frac{|u(x)| \eta(x)-t}{c(n)\left(\int_{\{|u| \eta>t\}} A(D[|u(y)| \eta(y)]) \mathrm{d} y\right)^{1 / n}}\right) \mathrm{d} x
\end{aligned}
$$




$$
\leqslant 2 k c(n)\left(\int_{\{|u| \eta>t\}} A(D[|u| \eta]) \mathrm{d} x\right)^{1+1 / n}<+\infty .
$$

Now, if

$$
\int^{+\infty}\left(\frac{s}{A_{\star}(s)}\right)^{n^{\prime}-1} \mathrm{~d} s<+\infty,
$$

then there exists $s_{0}>0$ such that $A_{n}(s)=+\infty$ for $s \geqslant s_{0}$. Hence, inequality (3.10) tells us that $|u| \leqslant\left(s_{0} / 2 k\right)+t$ in $B_{R}$.

If, on the contrary,

$$
\int^{+\infty}\left(\frac{s}{A_{\star}(s)}\right)^{n^{\prime}-1} \mathrm{~d} s=+\infty
$$

then $A_{n}$ is everywhere finite, so that the last two integrals in (3.8) are finite. Thus, inequality (3.7) follows from (3.8) and (3.10).

The Caccioppoli inequality for a local minimizer $u$ of $J$ is contained in the next lemma. In the statement, given any ball $B_{R} \subset \Omega$ and any $t \in \mathbb{R}, E_{t, R}$ will denote the set defined by

$$
E_{t, R}=\left\{x \in B_{R}: u(x)>t\right\} .
$$

LEMMA 3.-Let $A$ and $B$ satisfy assumptions (2.2)-(2.5). Let $F$ : $[0,+\infty) \rightarrow[0,+\infty)$ be an increasing function satisfying the $\Delta_{2}-$ condition. Suppose that a number $s_{0} \geqslant 0$ exists such that

$$
A(\xi)+B(|\xi|) \leqslant F(|\xi|) \text { for }|\xi| \geqslant s_{0}
$$

Let $u$ be any local minimizer of $J$. Then, given any positive numbers $\delta, R_{0}$ and $t_{1}$, there exists a constant $C>0$, depending only on $I(F), \delta, R_{0}, s_{0}$ and $t_{1}$, such that

$$
\begin{aligned}
\int_{E_{t, \rho}} A(D u) \mathrm{d} x \leqslant & C\left(\frac{1}{(R-\rho)^{I(F)+\delta}} \int_{E_{t, R}}(1+b(x)) F(u-t) \mathrm{d} x\right. \\
& \left.+F(t) \int_{E_{t, R}} b(x) \mathrm{d} x+\int_{E_{t, R}}(1+a(x)) \mathrm{d} x\right)
\end{aligned}
$$

for every $0<\rho<R \leqslant R_{0}$ and every $t \geqslant t_{1}$. 
Proof. - All the balls considered throughout the proof will be centered at a fixed point of $\Omega$. Moreover, all the constants will be allowed to depend on the same quantities as the constant $C$ in (3.13).

Let $\rho<\tau \leqslant \sigma \leqslant R \leqslant R_{0}$ and let $\eta$ be any real-valued smooth function on $\mathbb{R}^{n}$ such that $0 \leqslant \eta(x) \leqslant 1$ for every $x \in \mathbb{R}^{n}, \eta \equiv 1$ in $B_{\tau}, \eta \equiv 0$ outside $B_{\sigma}$ and $|D \eta| \leqslant 2 /(\sigma-\tau)$ everywhere. Fix any $t \geqslant t_{1}$ and choose

$$
\phi=-\eta^{I(F)+\delta}(u-t)_{+}
$$

in (2.8). Here, subscript + denotes positive part. Let us set $v=u+\phi$. Since $\phi$ vanishes outside $E_{t, \sigma}$, inequality (2.8) and assumption (2.1) imply that

$$
\begin{aligned}
\int_{E_{t, \sigma}} A(D u) \mathrm{d} x \leqslant & c_{1}\left(\int_{E_{t, \sigma}} A(D v) \mathrm{d} x+\int_{E_{t, \sigma}} b(x)[B(|u|)+B(|v|)] \mathrm{d} x\right. \\
& \left.+\int_{E_{t, \sigma}} a(x) \mathrm{d} x\right)
\end{aligned}
$$

for a suitable constant $c_{1}>0$. Consider the first integral on the right-hand side of (3.14). We have

$$
\begin{aligned}
D v= & \left(1-\eta^{I(F)+\delta}\right) D u \\
& +(I(F)+\delta) \eta^{I(F)+\delta} \frac{D \eta}{\eta}(t-u) \quad \text { for } x \in E_{t, \sigma} .
\end{aligned}
$$

By the convexity of $A$, by our assumptions on $\eta$ and by Proposition 1 applied to $A_{M}$, there exists a constant $c_{2}>0$ such that

$$
\begin{aligned}
\int_{E_{t, \sigma}} A(D v) \mathrm{d} x \leqslant & \int_{E_{t, \sigma}}\left(1-\eta^{I(F)+\delta}\right) A(D u) \mathrm{d} x \\
& +\int_{E_{t, \sigma}} \eta^{I(F)+\delta} A\left((I(F)+\delta) \frac{D \eta}{\eta}(t-u)\right) \mathrm{d} x \\
\leqslant & \int_{E_{t, \sigma} \backslash E_{t, \tau}} A(D u) \mathrm{d} x \\
& +c_{2} \int_{E_{t, \sigma}} A_{M}\left(\frac{2}{\sigma-\tau}(u-t)\right) \mathrm{d} x .
\end{aligned}
$$

On the other hand, assumption (3.12) and Proposition 1 ensure that 


$$
\begin{aligned}
& \int_{E_{t, \sigma}} A_{M}\left(\frac{2}{\sigma-\tau}(u-t)\right) \mathrm{d} x \\
& \leqslant \int_{E_{t, \sigma}} F\left(\frac{2}{\sigma-\tau}(u-t)\right) \mathrm{d} x+A_{M}\left(s_{0}\right)\left|E_{t, \sigma}\right| \\
& \leqslant \frac{c_{3}}{(\sigma-\tau)^{I(F)+\delta}} \int_{E_{t, \sigma}} F(u-t) \mathrm{d} x+A_{M}\left(s_{0}\right)\left|E_{t, \sigma}\right|
\end{aligned}
$$

for some positive constant $c_{3}$. Combining (3.15) and (3.16) yields

$$
\begin{aligned}
\int_{E_{t, \sigma}} A(D v) \mathrm{d} x \leqslant & \int_{\substack{E_{t, \sigma} \backslash E_{t, \tau}\\
}} A(D u) \mathrm{d} x+\frac{c_{2} c_{3}}{(\sigma-\tau)^{I(F)+\delta}} \int_{E_{t, \sigma}} F(u-t) \mathrm{d} x \\
& +c_{2} A_{M}\left(s_{0}\right)\left|E_{t, \sigma}\right| .
\end{aligned}
$$

Let us now take into account the second integral on the right-hand side of (3.14). Since $|u| \leqslant|u-t|+t$ and $|v| \leqslant|u|+|u-t| \leqslant t+2|u-t|$, we have by (3.12)

$$
\begin{aligned}
& \int_{E_{t, \sigma}} b(x)[B(|u|)+B(|v|)] \mathrm{d} x \\
& \leqslant 2 \int_{E_{t, \sigma}} b(x) B(t+2(u-t)) \mathrm{d} x \\
& \leqslant 2 \int_{E_{t, \sigma}} b(x) F(t+2(u-t)) \mathrm{d} x+2 B\left(s_{0}\right) \int_{E_{t, \sigma}} b(x) \mathrm{d} x \\
& \leqslant 2 \int_{E_{t, \sigma}} b(x) F(4(u-t)) \mathrm{d} x+2(F(2 t) \\
& \left.\quad+B\left(s_{0}\right)\right) \int_{E_{t, \sigma}} b(x) \mathrm{d} x .
\end{aligned}
$$

Hence, a constant $c_{4}>0$ exists such that

$$
\begin{aligned}
\int_{E_{t, \sigma}} b(x)[B(|u|)+B(|v|)] \mathrm{d} x \leqslant & c_{4}\left(\frac{1}{(\sigma-\tau)^{I(F)+\delta}} \int_{E_{t, \sigma}} b(x) F(u-t) \mathrm{d} x\right. \\
& \left.+F(t) \int_{E_{t, \sigma}} b(x) \mathrm{d} x\right) .
\end{aligned}
$$


From (3.14), (3.17) and (3.19) we deduce that

$$
\begin{aligned}
& \int_{E_{t, \tau}} A(D u) \mathrm{d} x \\
& \leqslant c_{5}\left(\int_{E_{t, \sigma} \backslash E_{t, \tau}} A(D u) \mathrm{d} x+\frac{1}{(\sigma-\tau)^{I(F)+\delta}} \int_{E_{t, R}}(1+b(x)) F(u-t) \mathrm{d} x\right. \\
& \left.\quad+F(t) \int_{E_{t, R}} b(x) \mathrm{d} x+\int_{E_{t, R}}(1+a(x)) \mathrm{d} x\right)
\end{aligned}
$$

for some $c_{5}>0$. Summing up the quantity $c_{5} \int_{E_{t \tau}} A(D u) \mathrm{d} x$ to both sides of (3.20), dividing through by $\left(1+c_{5}\right)$ and applying a standard iteration argument (see, e.g., Lemma 3.1, Chapter 5 of [8]) yield (3.13).

\section{PROOF OF THEOREM 2}

Our approach is related to that of $[5,7,10]$. By Remark 1 we may assume, without loss of generality, that

$$
A(\xi)=|\xi|^{i_{\infty}\left(A_{\star}\right)-r_{\varepsilon}} \quad \text { for small }|\xi|
$$

Here, $r_{\varepsilon}=\varepsilon$ or $r_{\varepsilon}=0$ according to whether $i_{\infty}\left(A_{\star}\right)>1$ or $i_{\infty}\left(A_{\star}\right)=1$, and $\varepsilon$ is a positive number to be chosen later. To begin with, we require that $\varepsilon$ is so small that

$$
i_{\infty}\left(A_{\star}\right)-r_{\varepsilon} \geqslant 1
$$

and

$$
\varepsilon<i_{\infty}\left(A_{M}+B\right)
$$

Set

$$
\gamma=\max \left\{\left(i_{\infty}\left(A_{\star}\right)-r_{\varepsilon}\right)^{*} / \beta^{\prime}, i_{\infty}\left(A_{M}+B\right)-\varepsilon\right\}
$$

and let $F:[0,+\infty) \rightarrow[0,+\infty)$ be any increasing function such that

$$
F(s)= \begin{cases}s^{\gamma} & \text { for small } s \\ A_{M}(s)+B(s) & \text { for large } s .\end{cases}
$$


Let us fix a ball $B_{R} \Subset \Omega$ with which every ball considered throughout the proof will be concentric. For every nonnegative integer $h$ we set

$$
\rho_{h}=\frac{R}{2}+\frac{R}{2^{h+1}}, \quad \bar{\rho}_{h}=\frac{\rho_{h}+\rho_{h+1}}{2}
$$

and, given $t \geqslant 1$,

$$
t_{h}=t\left(1-2^{-(h+1)}\right) .
$$

Clearly, $\rho_{h}$ decreases to $R / 2$ and $t_{h}$ increases to $t$ as $h$ goes to $+\infty$.

Let $u$ be any local minimizer of $J$. We set

$$
\begin{aligned}
J_{h}= & \int_{E_{t_{h}, \rho_{h}}}(1+b(x)) F\left(u-t_{h}\right) \mathrm{d} x \\
& +\int_{E_{t_{h}, \rho_{h}}}(1+a(x))\left(u-t_{h}\right)^{i_{\infty}\left(A_{\star}\right)+\varepsilon} \mathrm{d} x,
\end{aligned}
$$

where $E_{t_{h}, \rho_{h}}$ is defined as in (3.11). We claim that $J_{h}<+\infty$ for every $h$. Indeed, by Hölder's inequality,

$$
\begin{aligned}
& \int_{E_{t_{h}, \rho_{h}}}(1+b(x)) F\left(u-t_{h}\right) \mathrm{d} x \\
& \leqslant\left(\int_{E_{t_{h}, \rho_{h}}} F\left(u-t_{h}\right)^{\beta^{\prime}} \mathrm{d} x\right)^{1 / \beta^{\prime}}\left(\int_{E_{t_{h}, \rho_{h}}}(1+b(x))^{\beta} \mathrm{d} x\right)^{1 / \beta},
\end{aligned}
$$

with the usual modification if $\beta=\infty$. Owing to (4.1) and (4.4), there exists a constant $c_{1}$ such that $F(s)^{\beta^{\prime}} \leqslant c_{1} A_{n}(s)$ if $s$ is sufficiently small and $F(s)^{\beta^{\prime}}=\left(A_{M}(s)+B(s)\right)^{\beta^{\prime}}$ if $\mathrm{s}$ is sufficiently large. Hence, by (2.15), there exists $c_{2}>0$ such that

$$
F(s)^{\beta^{\prime}} \leqslant A_{n}\left(c_{2} s\right) \text { for } s \geqslant 0 .
$$

Thus, by Lemma 2 and assumption (i), the first integral on the right-hand side of (4.5) is finite. As far as the second integral is concerned, if $\varepsilon$ is so small that

$$
i_{\infty}\left(A_{\star}\right)+\varepsilon<\left(i_{\infty}\left(A_{\star}\right)-r_{\varepsilon}\right)^{*},
$$

then, by Hölder's inequality again, we have 


$$
\begin{aligned}
\int_{E_{t_{h}, \rho_{h}}} & (1+a(x))\left(u-t_{h}\right)^{i_{\infty}\left(A_{\star}\right)+\varepsilon} \mathrm{d} x \\
\leqslant & \left(\int_{E_{t_{h}, \rho_{h}}}\left(u-t_{h}\right)^{\left(i_{\infty}\left(A_{\star}\right)-r_{\varepsilon}\right)^{*}} \mathrm{~d} x\right)^{\frac{i_{\infty}\left(A_{\star}\right)+\varepsilon}{\left(i_{\infty}\left(A_{\star}\right)-r_{\varepsilon}\right)^{*}}} \\
& \times\left(\int_{E_{t_{h}, \rho_{h}}}(1+a(x))^{\left(\frac{\left(i \infty\left(A_{\star}\right)-r_{\varepsilon}\right)^{*}}{i_{\infty}\left(A_{\star}\right)+\varepsilon}\right)^{\prime}} \mathrm{d} x\right)^{1 /\left(\frac{\left(i_{\infty}\left(A_{\star}\right)-r_{\varepsilon}\right)^{*}}{i_{\infty}\left(A_{\star}\right)+\varepsilon}\right)^{\prime}} .
\end{aligned}
$$

Observe now that a positive constant $c_{3}$ exists such that

$$
s^{i_{\infty}\left(A_{\star}\right)-r_{\varepsilon}} \leqslant c_{3} A_{\star}(s) \text { for } s \geqslant 0 .
$$

Inequality (4.10) follows from (4.1) and Proposition 1 if $i_{\infty}\left(A_{\star}\right)>1$, and from (4.1) and the convexity of $A_{\star}$ if $i_{\infty}\left(A_{\star}\right)=1$. Inequality (4.10) implies that a constant $c_{4}>0$ exists such that

$$
s^{\left(i_{\infty}\left(A_{\star}\right)-r_{\varepsilon}\right)^{*}} \leqslant c_{4} A_{n}(s) \text { for } s \geqslant 0 .
$$

Thus, the first integral on the right-hand side of (4.9) is finite by Lemma 2. The second is also finite, provided that $\varepsilon$ is chosen so small that

$$
\left(\frac{\left(i_{\infty}\left(A_{\star}\right)-r_{\varepsilon}\right)^{*}}{i_{\infty}\left(A_{\star}\right)+\varepsilon}\right)^{\prime}<\alpha .
$$

Such a choice of $\varepsilon$ is possible thanks to assumption (ii). The finiteness of $J_{h}$ is proved.

Inequalities (4.6) and (4.9) ensure that, if $R_{0}$ is any positive number and $R \leqslant R_{0}$, then a constant $c_{5}$ exists such that

$$
\begin{aligned}
J_{h} \leqslant & c_{5}\left\{\left(\int_{E_{t_{h}, \rho_{h}}} F\left(u-t_{h}\right)^{\beta^{\prime}} \mathrm{d} x\right)^{1 / \beta^{\prime}}\right. \\
& \left.+\left(\int_{E_{t_{h}, \rho_{h}}}\left(u-t_{h}\right)^{\left(i_{\infty}\left(A_{\star}\right)-r_{\varepsilon}\right)^{*}} \mathrm{~d} x\right)^{\frac{i \infty\left(A_{\star}\right)+\varepsilon}{\left(i_{\infty}\left(A_{\star}\right)-r_{\varepsilon}\right)^{*}}}\right\}
\end{aligned}
$$

for every $h$. Fix now any smooth function $\omega:[0,+\infty) \rightarrow[0,1]$ such that $\omega(s)=1$ if $s \in[0,1 / 2], \omega(s)=0$ if $s \geqslant 3 / 4$ and $|\mathrm{d} \omega / \mathrm{d} s| \leqslant 4$ for every 
$s \in[0,+\infty)$. On setting

$$
\omega_{h}(x)=\omega\left(\frac{2^{h+1}}{R}\left(|x|-\frac{R}{2}\right)\right),
$$

one has $\omega_{h}=1$ in $B_{\rho_{h+1}}, \omega_{h}=0$ outside $B_{\bar{\rho}_{h}}$ and $\left|D \omega_{h}\right| \leqslant 2^{h+3} / R$ everywhere. Inequalities (4.13), (4.7), (4.11) and Lemma 1 yield

$$
\begin{aligned}
J_{h+1} \leqslant & c_{5}\left\{\left(\int_{E_{t_{h+1}, \bar{\rho}_{h}}} F\left(\left(u-t_{h+1}\right) \omega_{h}\right)^{\beta^{\prime}} \mathrm{d} x\right)^{1 / \beta^{\prime}}\right. \\
& \left.+\left(\int_{E_{t_{h+1}, \bar{\rho}_{h}}}\left(\left(u-t_{h+1}\right) \omega_{h}\right)^{\left(i_{\infty}\left(A_{\star}\right)-r_{\varepsilon}\right)^{*}} \mathrm{~d} x\right)^{\frac{i_{\infty}\left(A_{\star}\right)+\varepsilon}{\left(i_{\infty}\left(A_{\star}\right)-r_{\varepsilon}\right)^{*}}}\right\} \\
\leqslant & c_{6}\left\{\int_{E_{t_{h+1}, \bar{\rho}_{h}}} A\left(D\left[\left(u-t_{h+1}\right)_{+} \omega_{h}\right]\right) \mathrm{d} x\right. \\
& \left.\times \Psi\left(c_{c_{2} c(n)}\left(\int_{E_{t_{h+1}, \bar{\rho}_{h}}} A\left(D\left[\left(u-t_{h+1}\right)_{+} \omega_{h}\right]\right) \mathrm{d} x\right)^{1 / n}\right)\right\}^{1 / \beta^{\prime}} \\
& \left.+\left(\int_{E_{t_{h+1}, \bar{\rho}_{h}}} A\left(D\left[\left(u-t_{h+1}\right)_{+} \omega_{h}\right]\right) \mathrm{d} x\right)^{\frac{i \infty\left(A_{\star}\right)+\varepsilon}{i_{\infty}\left(A_{\star}\right)-r_{\varepsilon}}}\right\}
\end{aligned}
$$

for some $c_{6}>0$, where $\Psi:[0,+\infty) \rightarrow[0,+\infty)$ is any function such that $F^{\beta^{\prime}}(\lambda s) \leqslant \Psi(\lambda) F^{\beta^{\prime}}(s)$. Since $i_{\infty}\left(F^{\beta^{\prime}}\right)=\beta^{\prime} i_{\infty}(F)=\beta^{\prime} i_{\infty}\left(A_{M}+\right.$ $B), I\left(F^{\beta^{\prime}}\right)=\beta^{\prime} I(F)$ and $A_{M}+B$ satisfies the $\Delta_{2}$-condition, Eq. (4.4) and Proposition 2 below ensure that one can take

$$
\Psi(\lambda)=c_{7}\left(\lambda^{\beta^{\prime}\left(i_{\infty}\left(A_{M}+B\right)-\varepsilon\right)}+\lambda^{\beta^{\prime}(I(F)+\varepsilon)}\right)
$$

in (4.14), where $c_{7}$ is a suitable positive number. Notice now that

$$
\begin{aligned}
& \int_{E_{t_{h+1}, \bar{\rho}_{h}}} A\left(D\left[\left(u-t_{h+1}\right)_{+} \omega_{h}\right]\right) \mathrm{d} x \\
& \leqslant c_{8}\left(\int_{E_{t_{h+1}, \bar{\rho}_{h}}} A(D u) \mathrm{d} x+\int_{E_{t_{h+1}, \bar{\rho}_{h}}} A_{M}\left(2^{h}\left(u-t_{h+1}\right)\right) \mathrm{d} x\right)
\end{aligned}
$$


for some constant $c_{8}$ independent of $h$. One can make use of the Caccioppoli inequality (3.13) to estimate the former integral on the righthand side of (4.16), and exploit the fact that $A_{M}(s) \leqslant F(s)+c_{9}$ for $s \geqslant 0$ for some $c_{9}>0$ and Proposition 1 to estimate the latter. So doing, we deduce that, for every $\delta>0$, a constant $c_{10}$, independent of $h$, exists such that

$$
\begin{aligned}
& \int_{E_{t_{h+1}, \bar{\rho}_{h}}} A\left(D\left[\left(u-t_{h+1}\right)_{+} \omega_{h}\right]\right) \mathrm{d} x \\
& \leqslant c_{10}\left(2^{h(I(F)+\delta)} \int_{E_{t_{h+1}, \rho_{h}}}(1+b(x)) F\left(u-t_{h+1}\right) \mathrm{d} x\right. \\
& \left.+F(t) \int_{E_{t_{h+1}, \rho_{h}}} b(x) \mathrm{d} x+\int_{E_{t_{h+1}, \rho_{h}}}(1+a(x)) \mathrm{d} x\right) .
\end{aligned}
$$

We have

$$
J_{h} \geqslant \int_{E_{t_{h+1}, \rho_{h}}}(1+b(x)) F\left(u-t_{h+1}\right) \mathrm{d} x .
$$

Moreover,

$$
\begin{aligned}
J_{h} & \geqslant \int_{E_{t_{h+1}, \rho_{h}}}(1+b(x)) F\left(u-t_{h}\right) \mathrm{d} x \\
& \geqslant F\left(t_{h+1}-t_{h}\right) \int_{E_{t_{h+1}, \rho_{h}}}(1+b(x)) \mathrm{d} x \\
& =F\left(t 2^{-(h+2)}\right) \int_{E_{t_{h+1}, \rho_{h}}}(1+b(x)) \mathrm{d} x \\
& \geqslant c_{11} F(t) 2^{-(h+2)(I(F)+\delta)} \int_{E_{t_{h+1}, \rho_{h}}} b(x) \mathrm{d} x
\end{aligned}
$$

for some $c_{11}>0$. Similarly, since $t \geqslant 1$,

$$
\begin{aligned}
J_{h} & \geqslant \int_{E_{t_{h+1}, \rho_{h}}}(1+a(x))\left(u-t_{h}\right)^{i_{\infty}\left(A_{\star}\right)+\varepsilon} \mathrm{d} x \\
& \geqslant\left(t 2^{-(h+2)}\right)^{i_{\infty}\left(A_{\star}\right)+\varepsilon} \int_{E_{t_{h+1}, \rho_{h}}}(1+a(x)) \mathrm{d} x
\end{aligned}
$$




$$
\geqslant 2^{-(h+2)\left(i_{\infty}\left(A_{\star}\right)+\varepsilon\right)} \int_{E_{t_{h+1}, \rho_{h}}}(1+a(x)) \mathrm{d} x .
$$

From (4.17)-(4.20) we deduce that, if $\delta$ is sufficiently large, then

$$
\begin{aligned}
& \int_{E_{t_{h+1}, \bar{\rho}_{h}}} A\left(D\left[\left(u-t_{h+1}\right)_{+} \omega_{h}\right]\right) \mathrm{d} x \\
& \leqslant c_{12}\left(2^{h(I(F)+\delta)} J_{h}+2^{h\left(i_{\infty}\left(A_{\star}\right)+\varepsilon\right)} J_{h}\right) \\
& \leqslant c_{12} 2^{h(I(F)+\delta)} J_{h},
\end{aligned}
$$

where $c_{12}$ is a positive constant independent of $h$. Owing to (4.14), (4.15) and (4.21), one has

$$
\begin{aligned}
J_{h+1} \leqslant & c_{13}\left\{\left\{2 ^ { h ( I ( F ) + \delta ) } J _ { h } \left(\left[2^{h(I(F)+\delta)} J_{h}\right]^{\beta^{\prime}\left(i_{\infty}\left(A_{M}+B\right)-\varepsilon\right)}\right.\right.\right. \\
& \left.\left.+\left[2^{h(I(F)+\delta)} J_{h}\right]^{\beta^{\prime}(I(F)+\varepsilon)}\right)^{1 / n}\right\}^{1 / \beta^{\prime}}+\left(2^{h(I(F)+\delta)} J_{h}\right)^{\frac{i \infty\left(A_{\star}\right)+\varepsilon}{i_{\infty}\left(A_{\star}\right)-r_{\varepsilon}}} \\
\leqslant & c_{14} 2^{h d}\left\{J_{h}^{\frac{1}{\beta^{\prime}}+\frac{i_{\infty}\left(A_{M}+B\right)-\varepsilon}{n}}+J_{h}^{\frac{i \infty\left(A_{\star}\right)+\varepsilon}{i \infty\left(A_{\star}\right)-r_{\varepsilon}}}\right\}
\end{aligned}
$$

where $c_{13}, c_{14}$ are constants independent of $h$, and

$$
d=(I(F)+\delta) \max \left\{\frac{1}{\beta^{\prime}}+\frac{I(F)+\varepsilon}{n}, \frac{i_{\infty}\left(A_{\star}\right)+\varepsilon}{i_{\infty}\left(A_{\star}\right)-r_{\varepsilon}}\right\} .
$$

Notice that in (4.22) we have made use of the fact that $i_{\infty}\left(A_{M}+B\right)=$ $i_{\infty}(F) \leqslant I(F)$ and that $J_{h} \leqslant J_{0}$ for every $h$. Assumption (i) ensures that we can choose $\varepsilon$ so small that

$$
\frac{1}{\beta^{\prime}}+\frac{i_{\infty}\left(A_{M}+B\right)-\varepsilon}{n}>1 .
$$

Thus, if $\varepsilon$ is chosen so small that inequalities (4.2), (4.3), (4.8), (4.12) and (4.23) are satisfied, then (4.22) and the inequality $J_{h} \leqslant J_{0}$ again imply that

$$
J_{h+1} \leqslant c_{15} 2^{h d} J_{h}^{1+\theta},
$$

where $c_{15}$ is a constant independent of $h$, and $\theta$ is the (positive) number defined by

$$
\theta=\min \left\{\frac{i_{\infty}\left(A_{M}+B\right)-\varepsilon}{n}-\frac{1}{\beta}, \frac{\varepsilon+r_{\varepsilon}}{i_{\infty}\left(A_{\star}\right)-r_{\varepsilon}}\right\} .
$$


Now, if $\mathrm{t}$ is so large that $J_{0} \leqslant c_{15}^{-1 / \theta} 2^{-d / \theta^{2}}$, then, by Lemma 4.7, Chapter 2 of [14], $\lim _{h \rightarrow+\infty} J_{h}=0$, whence

$$
u \leqslant t \quad \text { in } B_{R / 2} \text {. }
$$

This proves that $u$ is locally bounded from above. The local boundedness from below follows from the fact that $-u$ is a local minimizer of the functional obtained on replacing $f(x, s, \xi)$ by $f(x,-s,-\xi)$, an integrand still satisfying (2.1).

PROPOSITION 2.- Let $\gamma$ and $s_{0}$ be positive numbers and let $\Phi$ : $(0,+\infty) \rightarrow(0,+\infty)$ be an increasing function such that $\Phi(s)=s^{\gamma}$ for $s \leqslant s_{0}$. Assume that

$$
I_{\infty}(\Phi)<+\infty \text { and } i_{\infty}(\Phi)-\delta \leqslant \gamma
$$

for some $\delta \in\left(0, i_{\infty}(\Phi)\right)$. Then $I(\Phi)<+\infty$ and there exists a constant $C>0$ such that

$$
\Phi(\lambda s) \leqslant C \max \left\{\lambda^{i_{\infty}(\Phi)-\delta}, \lambda^{I(\Phi)+\delta}\right\} \Phi(s) \text { for } \lambda, s>0 .
$$

Proof. - By Proposition 1 there exist positive numbers $c_{1}$ and $s_{1}$ such that $\Phi(2 s) \leqslant c_{1} \Phi(s)$ for $s \geqslant s_{1}$. On the other hand, $\Phi(2 s)=2^{\gamma} \Phi(s)$ for $s \leqslant s_{0} / 2$. Since $\Phi(2 s) \leqslant\left(2 / s_{0}\right)^{\gamma} \Phi\left(2 s_{1}\right) \Phi(s)$ if $s_{0} / 2<s<s_{1}$, then $\Phi$ satisfies the $\Delta_{2}$-condition, whence $I(\Phi)<+\infty$.

Consider now inequality (4.24). Proposition 1 ensures that there exists a constant $c_{1}>0$ such that

$$
\Phi(\lambda s) \leqslant c_{1} \lambda^{I(\Phi)+\delta} \Phi(s) \text { for } \lambda \geqslant 1, s>0 .
$$

Thus, it suffices to show that

$$
\Phi(\lambda s) \leqslant c_{2} \lambda^{i_{\infty}(\Phi)-\delta} \Phi(s) \text { for } 0<\lambda \leqslant 1, s<0,
$$

for some $c_{2}>0$. By Proposition 1, there exists $s_{2}>0$ such that (4.25) is true for $s \geqslant s_{2}$. Since $\gamma \geqslant i_{\infty}(\Phi)-\delta,(4.25)$ is trivially true (with $\left.c_{2}=1\right)$ also for $s \leqslant s_{0}$. It is then easily verified that (4.25) holds for every $s>0$.

\section{REFERENCES}

[1] Acerbi E., Fusco N., Partial regularity under anisotropic $(p, q)$ growth conditions, J. Differential Equations 107 (1994) 46-67. 
[2] Battacharya T., Leonetti F., $W^{2,2}$ regularity for weak solutions of elliptic systems with nonstandard growth, J. Math. Anal. Appl. 176 (1993) 224-234.

[3] Cianchi A., Boundedness of solutions to variational problems under general growth conditions, Comm. Partial Differential Equations 22 (1997) 1629-1646.

[4] Cianchi A., A fully anisotropic Sobolev inequality, Pacific Journal of Math., to appear.

[5] De Giorgi E., Sulla differenziabilità e l' analiticità delle estremali degli integrali multipli regolari, Mem. Accad. Sci. Torino (Classe di Sci. Mat. Fis. Nat.) 3 (3) (1957) 25-43

[6] Dall' Aglio A., Mascolo E., Papi G., Regularity for local minima of functionals with non standard growth conditions, Rend. Mat. 18 (7) (1998) 305-326.

[7] Fusco N., Sbordone C., Some remarks on the regularity of minima of anisotropic integrals, Comm. Partial Differential Equations 18 (1993) 153-167.

[8] Giaquinta M., Multiple Integrals in the Calculus of Variations and Nonlinear Elliptic Systems, Annals of Math. Studies, Vol. 105, Princeton Univ. Press, Princeton, 1983.

[9] Giaquinta M., Growth conditions and regularity, a counterexample, Manuscr. Math. 59 (1987) 245-248.

[10] Giaquinta M., Giusti E., Quasi-minima, Ann. Inst. H. Poincaré (Analyse non Linéaire) 1 (1984) 79-107.

[11] Giusti E., Direct Methods in the Calculus of Variations, Unione Matematica Italiana, Bologna, 1994.

[12] Min-Chung Hong, Some remarks on the minimizers of variational integrals with non standard growth conditions, Boll. Un. Mat. Ital. 6-A (1992) 91-101.

[13] Korolev A.G., On the boundedness of generalized solutions of elliptic differential equations with nonpower nonlinearities, Math. USSR Sbornik 66 (1990) 83-106.

[14] Ladyzenskaya O.A., Ural'ceva N.N., Linear and Quasilinear Elliptic Equations, Academic Press, New York, 1968.

[15] Leonetti F., Weak differentiability for solutions to nonlinear elliptic systems with $(p, q)$ growth conditions, Ann. Mat. Pura Appl. 162 (1992) 349-366.

[16] Lieberman G.M., The natural generalization of the natural conditions of Ladyzhenskaya and Ural'tseva for elliptic equations, Comm. Partial Differential Equations 16 (1991) 311-361.

[17] Marcellini P., Un exemple de solution discontinue d' un probléme variationnel dans le cas scalaire, Preprint, 1987.

[18] Marcellini P., Regularity of minimizers of integrals of the calculus of variations with non standard growth conditions, Arch. Rat. Mech. Anal. 105 (1989) 267-284.

[19] Marcellini P., Regularity and existence of solutions of elliptic equations with $p, q$ growth conditions, J. Differential Equations 90 (1991) 1-30.

[20] Marcellini P., Regularity for elliptic equations with general growth conditions, J. Differential Equations 105 (1993) 296-333.

[21] Mascolo E., Papi G., Local boundedness of minimizers of integrals of the calculus of variations, Ann. Mat. Pura Appl. 167 (1994) 323-339.

[22] Mascolo E., Papi G., Harnack inequality for minimizers of integral functionals with general growth conditions, Nonlinear Differential Equations Appl. 3 (1996) 232244.

[23] Moscariello G., Nania L., Hölder continuity of minimizers of functionals with nonstandard growth conditions, Ric. Mat. 40 (1991) 259-273. 
[24] Stroffolini B., Global boundedness of solutions of anisotropic variational problems, Boll. Un. Mat. Ital 5-A (1991) 345-352.

[25] Talenti G., Boundedness of minimizers, Hokkaido Math. J. 19 (1990) 259-279. 